\title{
Children and adolescents with negative social jet lag value
}

Keywords: Social jetlag; Daytime sleepiness; Cognitive performance; Sleep; Wake-up time

Abbrevations: SJL: Social Jet Lag; AP: Academic Performance; HDL: High-Density Lipoprotein

\section{Opinion}

The discrepancy between work (school) and free days can be described as 'social jetlag (SJL)', and SJL is the absolute difference in midpoints of sleep between work (school) nights and free nights. ${ }^{1}$ SJL refers to a mismatch between biological and social timing. ${ }^{2} \mathrm{SJL}$ is known to be associated with variable problems ${ }^{3-7}$ such as behavioral problems, daytime sleepiness, academic achievement, cognitive performance, metabolic risk and depression scores, especially among those who had a SJL of more than 1 hour. ${ }^{5}$ However, to my knowledge, few descriptions have been made on negative SJL values. In my recent study, ${ }^{8}$ to my surprise, problems on negative SJL (an earlier mid sleep time on free days compared to work (or school) days) have been found. In the current brief introductory opinion, I would like to raise the presumable unfavorable impact on children and adolescents with negative SJL values.

In order to determine the lifestyle habits including SJL significantly associated with self-reported academic performance (AP) in children in grades 5 to 12 (aged from 10 to 18 years) in Japan, a total of 2,114 completed questionnaires ${ }^{\mathrm{i}}$ were analyzed. ${ }^{8}$ In this study, children were categorized into five groups according to their SJLii distribution: -1 or less (SJL 1, n=19), more than -1 and 0 or less (SJL 2, $\mathrm{n}=452$ ), more than 0 and 1 or less (SJL 3, $\mathrm{n}=1082$ ), more than 1 and 2 or less (SJL 4, $\mathrm{n}=459$ ), more than 2 (SJL 5, $\mathrm{n}=102$ ). Consistent to former reports, ${ }^{3-7}$ this study ${ }^{8}$ showed that the mean self-reported AP of SJL 3 was

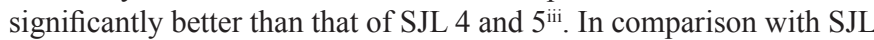
3 students, SJL 5 students were found to have a longer screen time ${ }^{\text {iv }}$,

iSelf-reported AP was selected from the following four categories after the general query of "what do you think of your own academic performance?"; 1. Good, 2. Relatively good, 3. Relatively poor, 4. Poor.

iiSJL was calculated from weekday bed time, weekend bed time, weekday wake-up time, and weekend wake-up time. Bed time and wake-up time were selected from the following nine categories divided by 1 hour; bed time (1. $<8 \mathrm{PM}, 2.8 \mathrm{PM}=<<9 \mathrm{PM}, 3.9 \mathrm{PM}=<<10 \mathrm{PM}, 4.10 \mathrm{PM}=<<11 \mathrm{PM}, 5.11 \mathrm{PM}=$ $<<0 \mathrm{AM}, 6.0 \mathrm{AM}=<<1 \mathrm{AM}, 7.1 \mathrm{AM}=<<2 \mathrm{AM}, 8.2 \mathrm{AM}=<<3 \mathrm{AM}, 9.3 \mathrm{AM}=$ $<$ ), wake-up time $(1 .<5 \mathrm{AM}, 2.5 \mathrm{AM}=<<6 \mathrm{AM}, 3.6 \mathrm{AM}=<<7 \mathrm{AM}, 4.7 \mathrm{AM}=$ $<<8 \mathrm{AM}, 5.8 \mathrm{AM}=<<9 \mathrm{AM}, 6.9 \mathrm{AM}=<<10 \mathrm{AM}, 7.10 \mathrm{AM}=<<11 \mathrm{AM}, 8$ $11 \mathrm{AM}=<<0 \mathrm{PM}, 9.0 \mathrm{PM}=<$ )

iiiANOVA was used to determine the differences between factors including self-reported AP and each SJL group. Student's $t$ test was used to assess the difference of factors between SJL subgroups.

${ }^{i v}$ On the screen time, the query was as follows; "How long do you use variable media tools such as TV, DVD, video, electrical game, PC, tablet, mobile phone, smartphone, etc.?" The selections were made by the following 5 groups; $1 .<2$ hours, 2. 2-4 hours, 3. 4-6 hours, 4. 6-8 hours, 5. 8 hours $=</$ day.
Volume I Issue 5 - 2017

\author{
Jun Kohyama \\ Tokyo Bay Urayasu Ichikawa Medical Center, Japan
}

Correspondence: Jun Kohyama, CEO, Tokyo Bay Urayasu Ichikawa Medical Center, 3-4-32 Toudaijima, Urayasu 279-000I, Japan,Email j-kohyama@jadecom.or.jp

Received: December 13, 2017 | Published: December 22, 2017

a later bed time, and a shorter weekday sleep duration, resulting in a later weekend wake-up time with a longer weekend sleep duration. In addition, breakfast and bowel habits of SJL 3 were better than that of SJL $5^{\mathrm{v}}$.

Interestingly, nearly a quarter $(22.3 \%$ (471/2114)) of whole students of the above-mentioned study ${ }^{8}$ was categorized into SJL 1 and 2, who had a SJL of 0 or less than 0 hours. Wong et al. [3] described that 68 out of 447 analyzed adult participants (15.2\%) had a SJL of less than 0 hours. However, they did not mention in details on these negative SJL value participants, although they found that greater SJL associated positively with triglycerides, fasting insulin, insulin resistance, waist circumference, and body mass index, and negatively with HDL-cholesterol.

The mean self-reported AP of SJL 1 in the above-mentioned study ${ }^{8}$ was significantly worse than that of SJL 3. In addition, compared with SJL 3 students, SJL 1 students showed a significantly higher male rate and had a shorter weekend sleep duration, a shorter weekend screen time, an earlier weekend bed time, a later weekday wake-up time, and an earlier weekend wake-up time. ${ }^{8}$ Moreover, SJL 1 students had a higher physical activity value than SJL 3 students. ${ }^{8}$ SJL 1 students in the study ${ }^{8}$ might be forced to wake up early in the weekend morning to practice physical activity, resulting in suffering from sleep loss and poor self-reported AP. Indeed, in my out-patient clinic, several students with complaints of daily sleepiness during school lesson confessed that they are active athletes and are obliged to join away matches almost every weekend, resulting in the shortage of sleep because of the many school obligations in addition to their practice as an athlete. I advised them to have a sleep during daily school lesson,

${ }^{v}$ Poor breakfast takers were those who rate 3 or 4 on the query of the frequency of taking breakfast (1. always, 2. often, 3. sometimes, 4. never). Poor bowel habits were defined as those who had constipation according to the standard criteria [Shih DQ, Kwan LY. All roads lead to Rome: Update on Rome III Criteria and new treatment options. Gastroenterol Rep 2007; 1: 56-65.], and those who rated 4 or more from the following selections were met to the definition of constipation (= poor bowel habits). The query on the defecation was as follows; 1 . Every day, 2. Every other day, 3. Once every two to three days, 4. twice a week, 5. Once a week, 6. More than a week interval. 
if they selected their priority on athlete's practice.

On SJL 2, their average self-reported AP was better than both SJL 4 and 5, but showed no significant difference with SJL $3 .{ }^{8}$ SJL value of -1 or less might be of negative influence on both mental and metabolic conditions.

\section{Acknowledgements}

None.

\section{Conflicts of interest}

The author declared that there are conflicts of interest.

\section{References}

1. Wittmann M, Dinich J, Merrow M, et al. Social jetlag: misalignment of biological and social time. Chronobiol Int. 2006;23(1-2):497-509.

2. Roenneberg T, Wirz-Justice A, Merrow M. Life between clocks: daily temporal patterns of human chronotypes. J Biol Rhythms. 2003;18(1):8090 .
3. Wong PM, Hasler BP, Kamarck TW, et al. Social jetlag, chronotype, and cardiometabolic risk. J Clin Endocrinol Metab. 2015;100(12):4612-4620.

4. Doi Y, Ishihara K, Uchiyama M. Associations of chronotype with social jetlag and behavioral problems in preschool children. Chronobiol Int. 2015;32(8):1101-1108.

5. Komada Y, Breugelmans R, Drake CL, et al. Social jetlag affects subjective daytime sleepiness in school-aged children and adolescents: A study using the Japanese version of the Pediatric Daytime Sleepiness Scale (PDSS-J). Chronobiol Int. 2016;33(10):1311-1319.

6. Díaz-Morales JF, Escribano C. Social jetlag, academic achievement and cognitive performance: Understanding gender/sex differences. Chronobiol Int. 2015;32(6):822-831.

7. Levandovski R, Dantas G, Fernandes LC, et al. Depression scores associate with chronotype and social jet lag in a rural population. Chronobiol Int. 2011;28(9):771-778.

8. Kohyama J. Self-reported academic performance and lifestyle habits of school children in Japan. Int J Child Health Nutrit. 2017;6(3):90-97. 